\title{
The influence of the energy of trauma, the timing of decompression, and the impact of grade of SCl on outcome
}

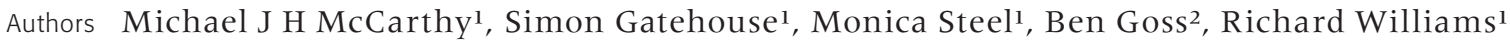 \\ Institution ${ }^{1}$ Princess Alexandra Hospital, Brisbane, Australia \\ ${ }^{2}$ Queensland University of Technology, Brisbane, Australia
}

\begin{tabular}{l|l|}
\hline $\begin{array}{l}\text { Final class of evidence } \\
\text { (CoE)-treatment }\end{array}$ & Yes \\
\hline Study design: & \\
\hline Prospective cohort & \\
\hline Retrospective cohort & \\
\hline Case control & \\
\hline Case series & \\
\hline Methods & \\
\hline Patients at similar point in & \\
\hline course of treatment & \\
\hline Follow-up $\geq 85 \%$ & \\
\hline Similarity of treatment & \\
\hline protocols for patient groups & \\
\hline Patients followed-up long & \\
enough for outcomes to occur & \\
\hline Control for extraneous & \\
\hline risk factors & \\
\hline Overall class of evidence & III \\
\hline The definiton of the different \\
\hline classes of evidence is available on \\
\hline page 55. \\
\hline
\end{tabular}

\section{ABSTRACT}

Study design: Retrospective cohort study.

Objectives: To find out: (1) if the energy of trauma (high and low) influence the outcome after cervical spinal cord injury; (2) if time to decompression and degree of injury (complete and incomplete) influence the outcome after high- and low-energy cervical spinal cord injury.

Methods: Twenty-one consecutive patients with low-energy cervical spinal cord injury were identified from the spinal injuries unit database (eg, ball sports, diving, surfing, and falls). Twenty-one aged-matched patients with high-energy cervical spinal cord injury (eg, motor vehicle trauma) were then randomly selected and the groups were compared. All patients had formal American Spinal Injuries Association assessment on admission and at 6 months.

Results: At the 6-month follow-up, the energy of the initial trauma was not found to influence the neurological outcome $(P=.76)$. Early definitive intervention ( $<8$ hours) for patients with incomplete cord lesions was shown to significantly affect outcome $(P=.049)$. As expected, patients with an incomplete spinal cord injury at presentation showed significantly greater neurological improvement at follow-up compared with those with complete injuries $(P=.006)$.

Conclusions: We were unable to find a correlation between the energy of the initial trauma causing a spinal cord injury and the neurological outcome. Early definitive decompression improved outcomes for patients with spinal cord injury, especially those with incomplete spinal cord injury. 


\section{STUDY RATIONALE AND CONTEXT}

Animal studies have shown that the rate, depth, and duration of spinal cord compression influence neurological prognosis [1-4]. The role of surgery in improving neurological recovery remains controversial. There is emerging clinical evidence that early decompression $(<24$ hours) improves neurological outcome [5-9]. Experimental evidence advocates decompression within 8 hours [10].

\section{OBJECTIVES}

To find out: (1) if the energy of trauma influence the outcome after cervical spinal cord injury (SCI); (2) if timing to definite decompression influence the outcome after cervical SCI.

\section{METHODS}

Study design: Retrospective cohort of patients treated for acute traumatic cervical SCI.

\section{Inclusion criteria:}

Low energy: acute traumatic cervical SCI from rugby, diving, surfing, and low-height falls.

High energy: acute traumatic cervical SCI from motor vehicle trauma.

Exclusion criteria: Central cord syndrome; no evidence of fracture or dislocation; coexisting significant head injury resulting in neurological impairment of the limbs. Nontraumatic causes of SCI/compression.

\section{Patient population and selection:}

The Princess Alexandra Hospital has an acute spinal cord trauma service and houses the Australian State of Queensland's SCI center on one campus.

- Over a 5-year period, 21 consecutive patients with low-energy cervical SCI admitted to the Spinal Injuries Unit were identified using the Spinal Injuries Unit Database.
- Patients for the low-energy cohort were age matched to within 3 years from the 139 cases of high-energy acute cervical traumatic SCI patients admitted during the same period. In cases of more than one appropriate match the high-energy cohort patient was selected using the "names from a hat" technique.

- All the patients underwent intervention to reduce, stabilize, and decompress the spinal cord either surgically or through traction and halo-vest immobilization.

\section{Outcomes and prognostic (risk) factors to be evaluated:}

- The American Spinal Injuries Association (ASIA) grade at presentation and at 6 months was measured on all patients. Complete SCI was defined as ASIA grade A (no motor or sensory function at S4/5) and incomplete as ASIA grades B to D (some motor or sensory function below the injury level).

- Neurological outcome was assessed by comparing the proportions that improved 1 or more ASIA grade between initial presentation and 6-month follow-up.

- Effect of time to definitive surgical intervention on ASIA grade: Early surgery was defined as within 8 hours of injury. Paramedic, hospital emergency department admission, and operating room patienttracking databases were used to accurately evaluate the time to surgery.

- The effect of low- versus high-energy injury on ASIA grade: Energy of injury was defined by mechanism. Low-energy injury was defined as those resulting from ball sports (eg, rugby, surfing, diving into shallow water, and falls from a standing height). Highenergy injury was defined as those resulting from motor vehicle trauma in which the kinetic energy was deemed to be far greater.

Analysis: The proportion that improved 1 or more ASIA grade between initial presentation and 6-month follow up was compared between cohorts using a Fisher's exact test.

- The cohorts comprised ASIA grade on presentation (complete or incomplete), time to surgery (early or late), and energy of injury (high or low). 


\section{RESULTS}

- Fig 1 shows the number of patients in each group.

- Table 1 shows patient demographics.

- Twenty-three patients had incomplete SCI and 19 had a complete SCI.

- Seventeen patients had early (<8 hours) and 25 had late intervention.

- Twenty-eight patients had anterior stabilization; 11 had nonsurgical decompression and stabilization; 2 had a combined anterior/posterior fixation; and 1 had posterior stabilization.

- The energy of injury did not influence neurological outcome $(P=.76$; Fig 2).
- The initial severity of injury (complete vs incomplete) was found to predict neurological outcome $(P=.006$; Fig 3).

- Table 2 displays results of the statistical analysis of the main groups.

- The energy of the injury in patients with an incomplete SCI did not influence neurological outcome ( $P=.73$; Fig 4).

- Time to definitive decompression ( $<8$ hours) in patients with incomplete SCI significantly influenced neurological outcome $(P=.049$; Fig 5).

- Tables 3 and 4 present results of the statistical analysis of the various subgroup analyses.

Fig 1 Patient numbers in each group used for analysis.

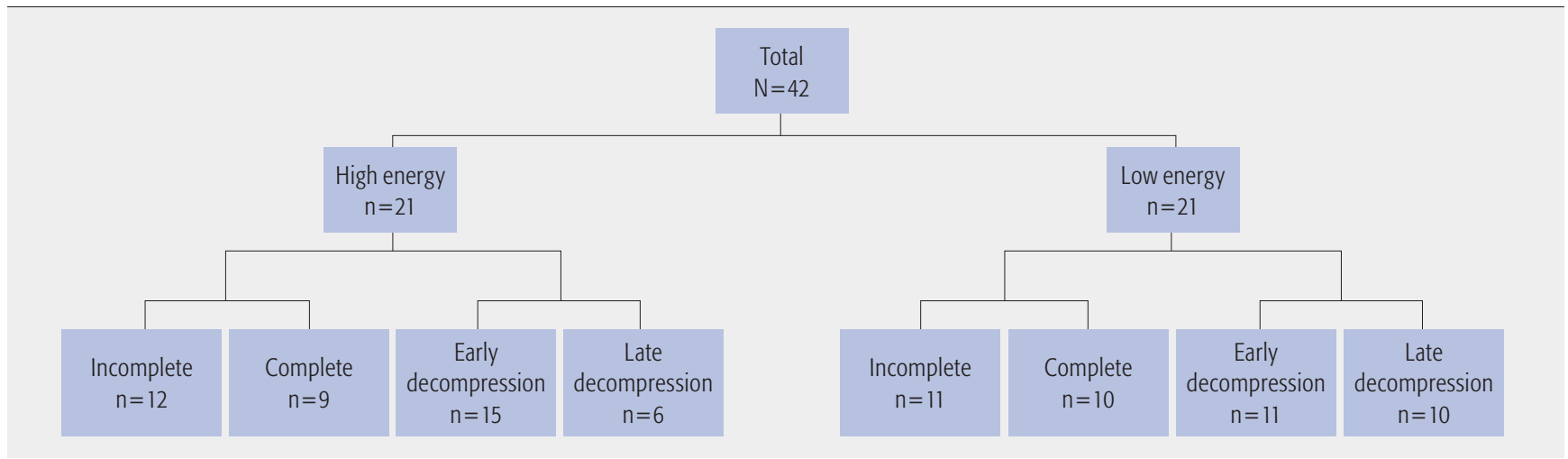

Fig 2 Functional recovery of 1 or more American Spinal Injuries Association grades in the study cohort as a function of the energy of the injury.

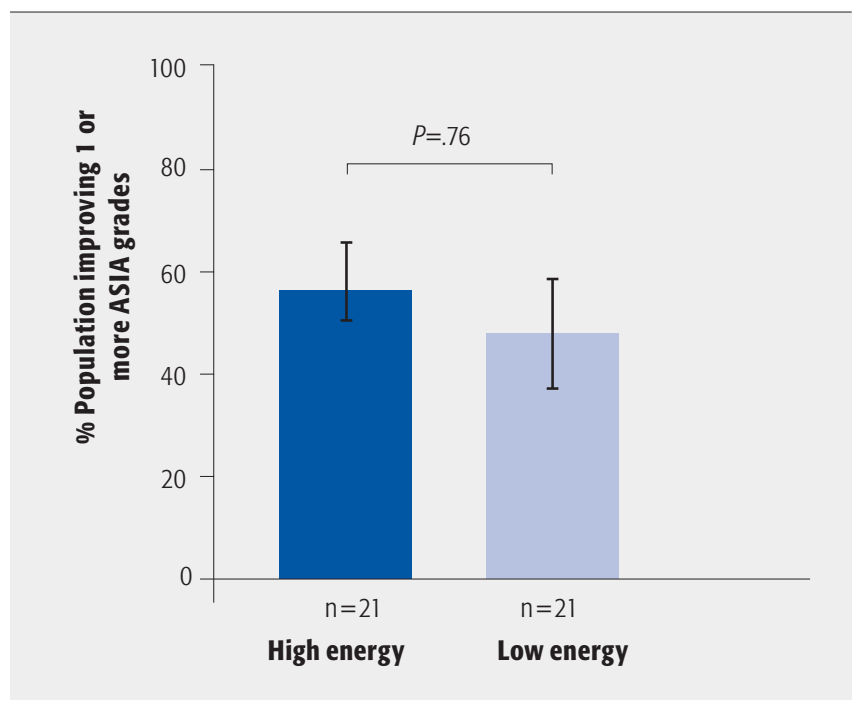

Fig 3 Functional recovery of 1 or more American Spinal Injuries Association grades in the study cohort as a function of the severity of the injury.

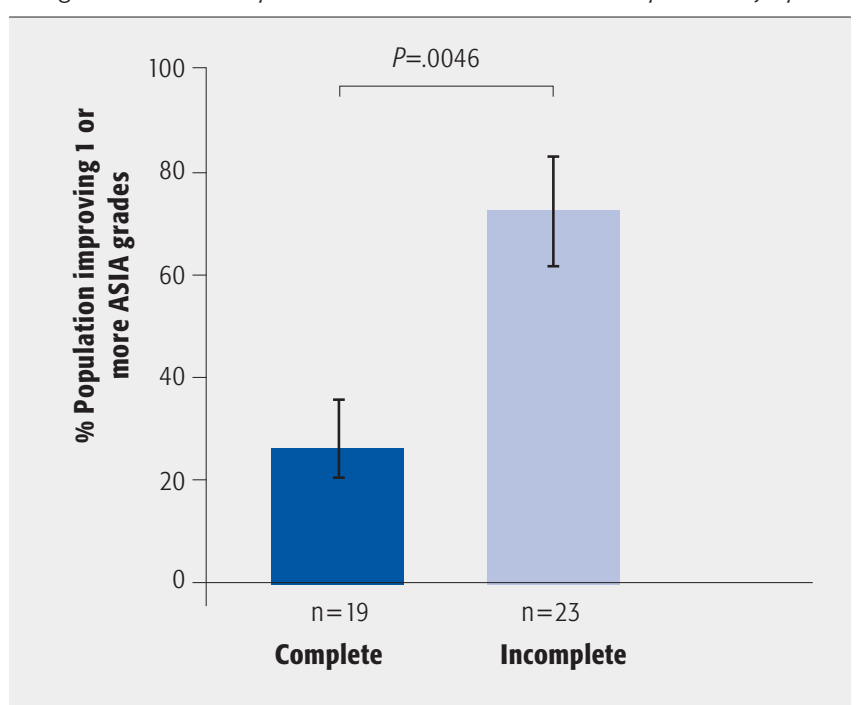


Fig 4 Functional recovery of 1 or more American Spinal Injuries Association grades in the incomplete group as a function of the energy of the injury.

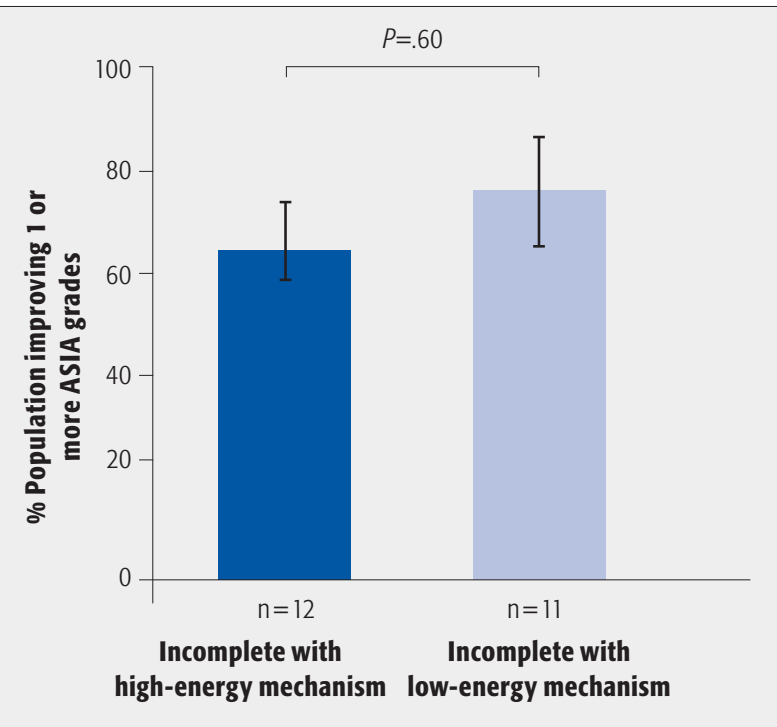

Table 1 Patient demographics.

\begin{tabular}{lll}
\hline Patient characteristics & Low energy & High energy \\
\hline $\mathrm{N}$ & 21 & 21 \\
\hline Age & Age matched & \\
\hline Female, \% & 4.7 & 47.0 \\
\hline Injury level & \\
\hline C3 & 1 & 1 \\
\hline C4 & 6 & 3 \\
\hline C5 & 11 & 9 \\
\hline C6 & 1 & 6 \\
\hline C7 & 1 & 2 \\
\hline Biomechanically stable injury & 4 & 6 \\
\hline Fracture & 3 & 18 \\
\hline Dislocation & 18 & 3 \\
\hline
\end{tabular}

Fig 5 Functional recovery of 1 or more American Spinal Injuries Association grades in the incomplete group as a function of the time to definitive treatment of the injury.

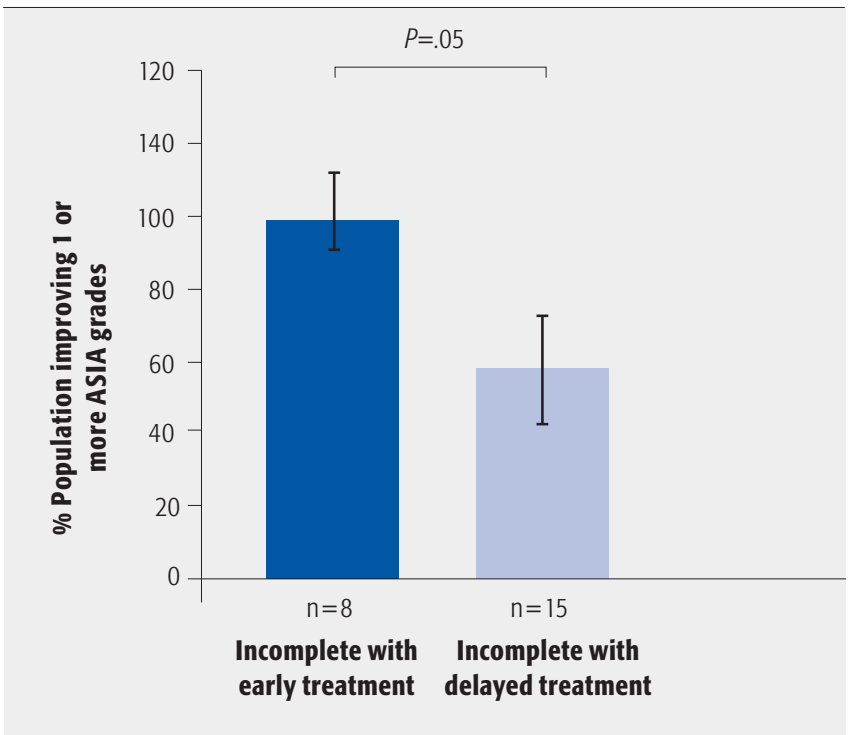

Table 2 Main analysis: proportion improving 1 or more ASIA grade.*

\begin{tabular}{|c|c|c|c|}
\hline $\begin{array}{l}\text { High energy } \\
\mathrm{n} / \mathrm{N}(\%)\end{array}$ & $\begin{array}{l}\text { Low energy } \\
\text { n/N (\%) }\end{array}$ & RR $(95 \% \mathrm{CI})$ & $\boldsymbol{P}$ \\
\hline 10/21 (47.6) & $12 / 21(57.1)$ & $1.2(0.67-2.15)$ & .76 \\
\hline $\begin{array}{l}\text { Incomplete } \\
\mathrm{n} / \mathrm{N}(\%)\end{array}$ & $\begin{array}{l}\text { Complete } \\
\text { n/N (\%) }\end{array}$ & RR $(95 \% \mathrm{CI})$ & $\boldsymbol{P}$ \\
\hline 17/23 (73.9) & $5 / 19(26.3)$ & $2.8(1.27-6.20)$ & .0046 \\
\hline Early n/N (\%) & Late $\mathrm{n} / \mathrm{N}(\%)$ & RR (95\% CI) & $P$ \\
\hline 9/17 (52.9) & $10 / 25(40.0)$ & $1.32(0.69-2.55)$ & .54 \\
\hline
\end{tabular}

*ASIA indicates American Spinal Injuries Association; RR, relative risk; and $\mathrm{Cl}$, confidence interval. 


\section{DISCUSSION}

McDonald and Sadowsky [8] suggested that subacute intervention (24-72 hours) has yielded unsatisfactory results because most tissue damage is irreversible by that time and suggested that trials were needed to assess the effects of early surgical intervention ( $<8$ hours).

Early results of the Surgical Treatment of Acute Spinal Cord Injury Study on the timing of surgery for spinal cord trauma indicate improved neurological outcome at 6- and 12-month follow-up in both patient groups ASIA A/B and ASIA C/D/E when treated by reduction and/or stabilization within 24 hours of injury compared with those treated later. This multicenter prospective nonrandomized study demonstrated that the early treatment group had a significantly larger percentage of patients improving 1 or more ASIA grades $(67 \%)$ compared with the late group $(40 \%)(P<.05)[5,7]$.

The results of our study confirm that the depth of neurological injury is the most consistent prognostic indicator in SCI. Findings would also suggest that early surgical management of incomplete SCI has a positive influence on functional outcome at 6 months (Table 3).

Logically, it could be postulated that prognosis following early reduction of lower-energy injuries may be more favorable than after higher-energy trauma. In the latter group, primary cord trauma is believed to cause a greater level of intrinsic cord disruption; whereas in the lowerenergy group, ongoing compression might be said to be the main contributor to neurological deficit, as there is a greater case for early reduction in the latter group. These results did not support this hypothesis, since there is no significant difference between the high- and low-energy groups in terms of timing of definitive management. Although neurological outcome was more favorable following early reduction of bifacetal dislocation, as has been previously established by other authors, the data would not suggest that energy or mechanism of injury has a positive influence on ASIA score at 6 months after injury (Table 4).

An incomplete SCI would appear to reflect a more modest primary injury to the cord despite the apparent level of energy associated with the injury. Our findings therefore do not support the concept of rate of injury, as outlined in animal studies, as a significant influence [1-4].

This study has a number of strengths and weaknesses. The data came from a single-site study database with reliable prospective data collection relating to the timing of surgery and ASIA score. To our knowledge, this is the first clinical study to consider energy of injury with a unique population base due to recreational and social demographics. It is however a small cohort and the follow-up time was limited. It is known that patients with SCI continue to show improvement up to and beyond 2 years following injury but most recovery occurs within the first 6 months.

While there was no significant difference in neurological outcome in the subset of patients sustaining SCI by lowvelocity mechanism $(P=.76)$, this study showed that more favorable prognosis in injuries associated with incomplete neurological deficit $(P=.006)$. Also patients with incomplete SCI treated definitively within 8 hours had a greater improvement in ASIA score compared with those treated after 8 hours $(P=.049)$. We would recommend early realignment for patients presenting with incomplete neurological deficit resulting from SCI.

Table 3 Subgroup analysis based on injury severity showing proportion improving 1 or more ASIA grades.*

\begin{tabular}{|c|c|c|c|c|c|c|c|}
\hline \multicolumn{4}{|c|}{ Incomplete } & \multicolumn{4}{|c|}{ Complete } \\
\hline High energy, $n / N$ & Low energy, $n / N$ & RR (95\% Cl) & $\boldsymbol{P}$ & High energy, $n / N$ & Low energy, $n / N$ & RR (95\% Cl) & $\boldsymbol{P}$ \\
\hline 9/11 (81.8\%) & $8 / 12(66.6 \%)$ & $1.22(0.75-1.99)$ & .60 & $4 / 9(44.4 \%)$ & $1 / 10(10.0 \%)$ & $2.24(0.97-5.12)$ & .14 \\
\hline Early, n/N & Late, $\mathrm{n} / \mathrm{N}$ & & & Early, $n / N$ & Late, $\mathrm{n} / \mathrm{N}$ & & \\
\hline $8 / 8(100 \%)$ & 8/15 (53.3\%) & $1.88(1.17-3.01)$ & .05 & $2 / 9(22.2 \%)$ & $3 / 10(30.0 \%)$ & $0.80(0.24-2.64)$ & 1.00 \\
\hline
\end{tabular}

*ASIA indicates American Spinal Injuries Association; RR, relative risk; and Cl, confidence interval.

Table 4 Subgroup analysis based on injury severity showing proportion improving 1 or more ASIA grades.*

\begin{tabular}{|c|c|c|c|c|c|c|c|}
\hline \multicolumn{4}{|c|}{ High energy } & \multicolumn{4}{|c|}{ Low energy } \\
\hline Early, n/N & Late, $\mathbf{n} / \mathbf{N}$ & RR $(95 \% \mathrm{CI})$ & $P$ & Early, $n / N$ & Late, n/N & RR (95\% CI) & $P$ \\
\hline $3 / 5(50.0 \%)$ & $7 / 15(46.6 \%)$ & $1.40(0.48-4.08)$ & .68 & $8 / 11(72.7 \%)$ & $3 / 10(30.0 \%)$ & $1.48(0.50-4.36)$ & .086 \\
\hline
\end{tabular}

*ASIA indicates American Spinal Injuries Association; RR, relative risk; and Cl, confidence interval. 
Clinical relevance and impact:

Supports the role of early intervention in improving neurological recovery.

\section{Future research:}

The Surgical Treatment of Acute Spinal Cord Injury Study (STASCIS Trial) is currently underway and will hopefully provide some definitive answers to the controversy surrounding the role of surgery in acute SCI.

\section{SUMMARY}

- We were unable to find a correlation between the energy of the initial trauma causing SCI and the neurological outcome.

- The initial severity of injury (complete vs incomplete) predicts neurological outcome.

- Early intervention (<8 hours) appears to improve the neurological outcome for patients with incomplete SCI.

\section{REFERENCES}

1. Delamarter RB, Sherman J, Carr JB (1995) Pathophysiology of spinal cord injury: recovery after immediate and delayed decompression. J Bone Joint Surg Am; 77(7):1042-1049.

2. Dimar JR II, Glassman SD, Raque GH, et al (1999) The influence of spinal canal narrowing and timing of decompression on neurologic recovery after spinal cord contusion in a rat model. Spine; 24(16):1623-1633.

3. Kearney PA, Ridella SA, Viano DC, et al (1988) Interaction of contact velocity and cord compression in determining the severity of spinal cord injury. J Neurotrauma; 5(3):187-208.

4. Noyes DH (1987) Correlation between parameters of spinal cord impact and resultant injury. Exp Neurol; 95(3): 535-547.

5. Arnold P (2008) S.T.A.S.C.I.S. Evaluating The Timing of Surgery in Cervical Spine Injury. Paper presented at: 23rd Annual Meeting of the Federation of Spine Associations; March 9, 2008, San Francisco, California.

6. Fehlings MG, Tator CH (1999) An evidencebased review of decompressive surgery in acute spinal cord injury: rationale, indications, and timing based on experimental and clinical studies. J Neurosurg; 91(1):1-11.

7. Fehlings MG, Wilson JR (2010) Timing of surgical intervention in spinal trauma: what does the evidence indicate? Spine; 35(21):159-160.

8. McDonald JW, Sadowsky C (2002) Spinalcord injury. Lancet; 359(9304):417-425.

9. McKinley W, Meade MA, Kirshblum S, et al (2004) Outcomes of early surgical management versus late or no surgical intervention after acute spinal cord injury. Arch Phys Med Rehabil; 85(11):1818-1825.

10. Assenmacher DR, Ducker TB (1971) Experimental traumatic paraplegia: the vascular and pathological changes seen in reversible and irreversible spinal-cord lesions. J Bone Joint Surg Am; 53(4):671-680. 


\section{EDITORIAL STAFF PERSPECTIVE}

The reviewers congratulated McCarthy and colleagues for introducing the element of injury energy into the scientific evaluation of SCI prognosis and care. The influence of kinetic energy on outcome is indeed an often overlooked factor. Setting the bar to canal decompression much higher than previously done by others by using a cutoff of 8 hours is a step in the right direction, and much more consistent with our understanding of largely irreversible temporal physiological changes that affect the injured cord. This study also supports the findings that injury severity is a profound determinant of eventual outcome.

While these considerations were seen very favorably, the reviewers also identified a number of concerns:

- SCI is a complex multifactorial entity. Covariables, such as preexistent stenosis, injury type (unilateral versus bilateral facet dislocation-type injuries, hyperextension injuries, burst fractures, shear injuries); injury severity scores affecting other organ systems; age of patient; restoration of normotension and quality of resuscitation; type of decompression and realignment/stabilization techniques used, are just some of the many factors which plausibly will impact patient outcomes.

- The actual differentiation of high-and low-energy injury based on described mechanisms is somewhat arbitrary as well.

- From a statistical point of view this study is most likely underpowered and suffers from some of the typical protocol inconsistencies, which decrease the evidence level of retrospective studies. For instance, McCarthy et al identified that seven patients had traction and four had external immobilization (which is a quarter of the 42-patient cohort). It is likely that these are the injuries that they referred to as 'biomechanically stable' - but they clearly represent a subpopulation that is widely different from patients with clearly unstable and displaced injuries, such as patients with complex fracture dislocations who required combined anterior and posterior procedures. From a methodological standpoint this subentity would preferably be assessed either separately or the main cohorts should be reassessed to make sure that they are similarly represented.
- Incomplete injuries have confounded many an SCI study as they represent a complex subentity, especially as far as central cord injuries are concerned. It has become increasingly clear that central cord syndromes are some of the injuries with better recovery potential. Yet again, this represents an injury entity which may heavily influence outcomes in observation cohorts and thus has to be accounted for separately to assure absence of observation bias.

- This study — and the factors raised in this discussion - again underscores the magnitude of difficulty individual SCI researchers face. From a statistical angle any study less than 1,000 is likely underpowered. Consistent treatment and assessment protocols applied over several years are really what are necessary to allow for larger scale conclusions on SCI care to be drawn.

In the big picture McCarthy et al deserve praise for investigating important and previously not discussed variables that influence SCI outcomes. Much hope and anticipation will be placed on the results of the STASCIS group and its unprecedented number of SCI patients treated in many reputable institutions. 